\title{
Macroenvironmental Regulation of Hair Cycling and Collective Regenerative Behavior
}

\author{
Maksim V. Plikus ${ }^{1}$ and Cheng-Ming Chuong ${ }^{2}$ \\ ${ }^{1}$ Department of Developmental and Cell Biology, Sue and Bill Gross Stem Cell Research Center, \\ University of California, Irvine, Irvine, California 92697 \\ ${ }^{2}$ Department of Pathology, Keck School of Medicine, University of Southern California, Los Angeles, \\ California 90033 \\ Correspondence: cmchuong@usc.edu
}

\begin{abstract}
The hair follicle (HF) regeneration paradigm provides a unique opportunity for studying the collective behavior of stem cells in living animals. Activation of HF stem cells depends on the core inhibitory BMPand activating WNT signals operating within the HF microenvironment. Additionally, HFs receive multilayered signaling inputs from the extrafollicular macroenvironment, which includes dermis, adipocytes, neighboring HFs, hormones, and external stimuli. These activators/inhibitors are integrated across multiple stem-cell niches to produce dynamic hair growth patterns. Because of their pigmentation, these patterns can be easily studied on live shaved animals. Comparing to autonomous regeneration of one HF, populations of HFs display coupled decision making, allowing for more robust and adaptable regenerative behavior to occur collectively. The generic cellular automata model used to simulate coordinated HF cycling here can be extended to study population-level behavior of other complex biological systems made of cycling elements.
\end{abstract}

$\mathrm{H}$ omeostasis of many adult organs largely depends on activities of tissue-specific stem cells (SCs). A lot of research has been focused on understanding how individual SCs or small clusters of SCs respond to signaling inputs from their immediate microenvironment, or the so-called niche, and decide between remaining quiescent or undergoing proliferation. This research yielded valuable insights into the mechanisms of regeneration and identified new therapeutic possibilities for tissue repair and replacement (Blanpain et al. 2004). However, despite significant advances in the field of SC biology, until recently relatively little was known about the mechanisms of organ-wide coordination among thousands of individual SCs.

In this respect, it is important to understand the principles of large-scale coupling among many dispersed SCs. It is reasonable to expect that any such principle would be universal, largely independent of the physical size or specific anatomical modifications of an organ. It is equally important to understand what signaling pathways mediate organ-wide SC coupling. Learning mechanisms of SC coordination will provide insights into the functional physiology of complex biological systems, beyond regeneration.

Editors: Anthony E. Oro and Fiona M. Watt

Additional Perspectives on The Skin and Its Diseases available at www.perspectivesinmedicine.org

Copyright (C) 2014 Cold Spring Harbor Laboratory Press; all rights reserved; doi: 10.1101/cshperspect.a015198

Cite this article as Cold Spring Harb Perspect Med 2014;4:a015198 
M.V. Plikus and C.-M. Chuong

Studying activities of thousands of SCs at the same time poses some unique challenges. Normally, powerful experimental techniques such as gene expression profiling on fluorescence-activated cell sorting (FACS) purified cells are not practical for this purpose. The usefulness of other techniques that account for the microanatomy of SC niche, such as conventional immunohistochemistry, is limited by the tissue sample size and inability to monitor changes in the same sample over time. An ideal experimental approach should enable noninvasive in vivo monitoring, recording, and analysis of activities in a very large population of SCs. For this approach to work, the physical location of SCs within an experimental system should be known and the output of SC activities should be easily observable. The majority of actively regenerating mammalian tissues and organs, however, fail to satisfy the above requirements. Here, we will show how various features of a hair follicle (HF) uniquely position it to serve as a powerful experimental system for learning about the mechanisms of coordinated regenerative behavior in a very large population of adult SCs.

\section{HF AS THE UNIQUE MODEL FOR STUDYING CYCLIC REGENERATION OF A SINGLE STEM-CELL CLUSTER AND THE COORDINATED REGENERATION IN A LARGE AND HETEROGENEOUS STEM-CELL POPULATION}

HF is an epithelio-mesenchymal miniorgan of the skin that periodically makes new hair shaft during the repetitive regeneration process known as the hair growth cycle (Müller-Rover et al. 2001; Stenn and Paus 2001). To sustain cyclic regeneration, each HF relies on its epithelial SCs. Diverse epithelial SC types populate anatomically discrete compartments in the upper portion of the HF: bulge, hair germ, isthmus, and infundibulum (Arwert et al. 2012). Among these SCs, activation of bulge SCs and their immediate progenies in the so-called hair germ occurs at the end of the resting phase of the cycle (telogen) (Cotsarelis et al. 1990; Morris et al. 2004; Tumbar et al. 2004). To a large extent, this activation event is driven by proproliferative signals from the specialized mesenchymal cells of dermal papilla (DP) (Greco et al. 2009; Enshell-Seijffers et al. 2010). Once SCs become activated, HF enters into the growth phase of the cycle (anagen). During anagen hair germ and bulge SCs give rise to transiently amplifying cells of the so-called epithelial matrix at the base of the HF. DP that becomes surrounded by matrix cells stimulates them to continuously proliferate and differentiate toward new hair shafts and inner root sheaths. Once a new hair shaft grows out to a certain length, matrix cells undergo apoptosis, effectively pushing the HF into the involution phase of the cycle (catagen). By the end of catagen the HF remodels back into its resting phase state, completing the cycle (Fig. 1A).

Skin contains thousands of HFs, each with its own cluster of bulge SCs. All HFs are distributed on the skin surface in a nearly geometrically precise gridlike pattern established early on during embryonic development (Fig. 1B) (Sick et al. 2006). As all HFs undergo regeneration, their growth cycle phases become coordinated across the two-dimensional space of the skin, so that large populations of HFs either remain in telogen or enter new anagen simultaneously. In many species including mice, rats, hamsters, chinchillas, and rabbits such coordinated regenerative behavior can be easily visualized as dynamic skin pigmentation patterns (Fig. 1C,D) (Durward and Rudall 1949; Whiteley and Ghadially 1954; Chase and Eaton 1959). These pigmentation patterns form because of the phenomenon of "anagen-coupled melanogenesis." Pigment production in mouse skin occurs exclusively in a matrix of anagen HFs. Some HFs in the telogen phase, as well as the skin between HFs, are not pigmented. Additionally, pigmentation of anagen HFs becomes visible a few days after the key SC activation event at the onset of each new hair growth cycle (Fig. 1E). Therefore, macroscopic pigmentation patterns can be used as an efficient noninvasive readout of skin-wide patterns of HF SC activation events that happened just a few days earlier (Ma et al. 2003; Suzuki et al. 2003; Plikus and Chuong 2008; Plikus et al. 2008). 
A

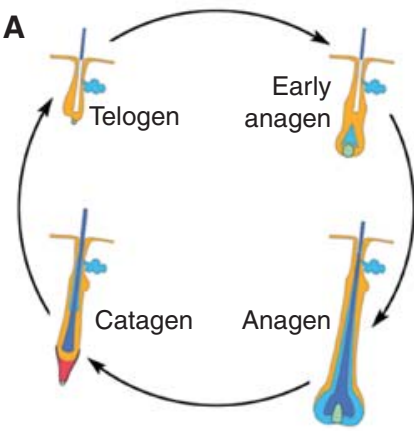

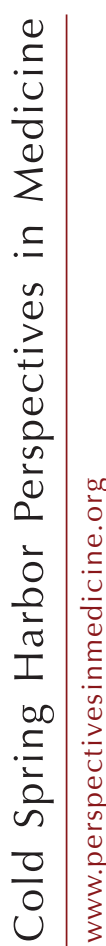
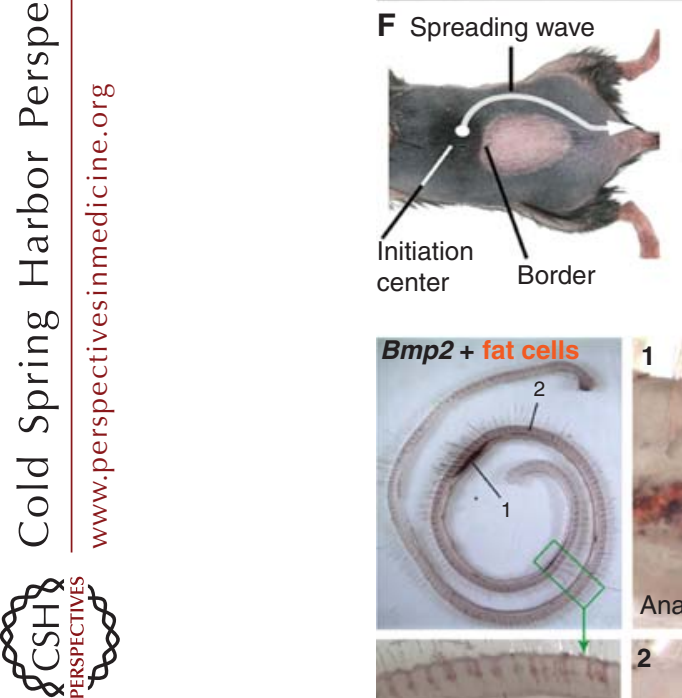

\section{G}

center
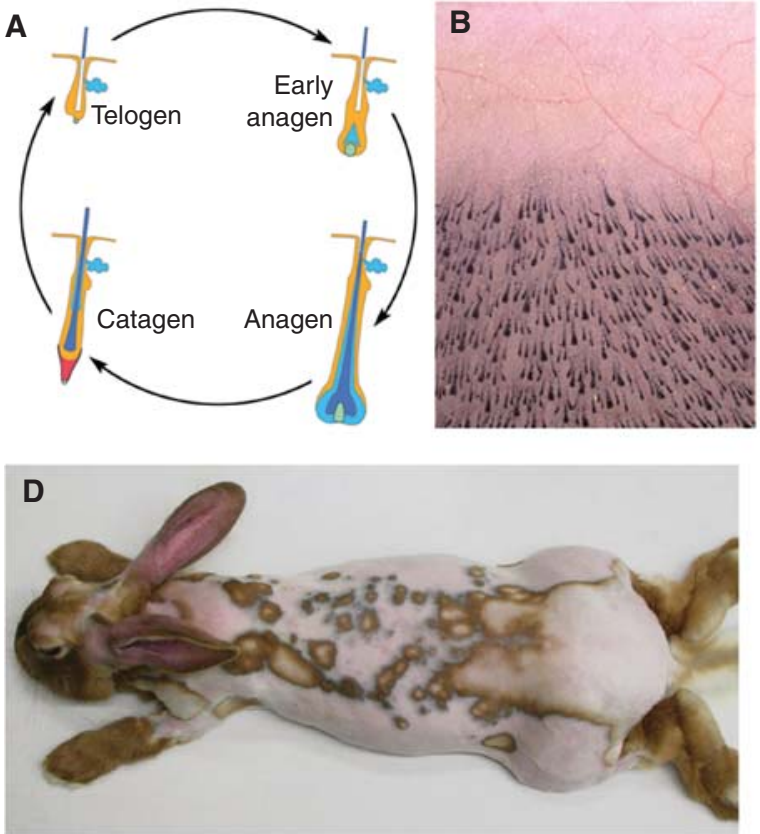

C

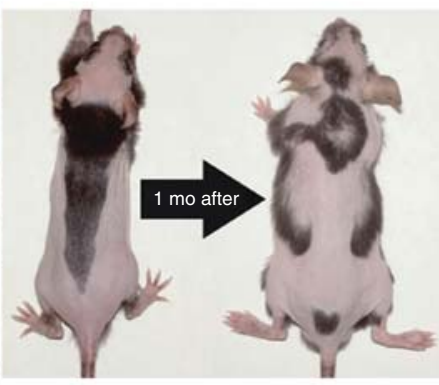

E

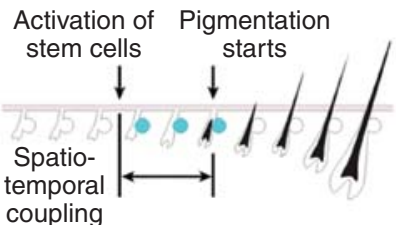

coupling
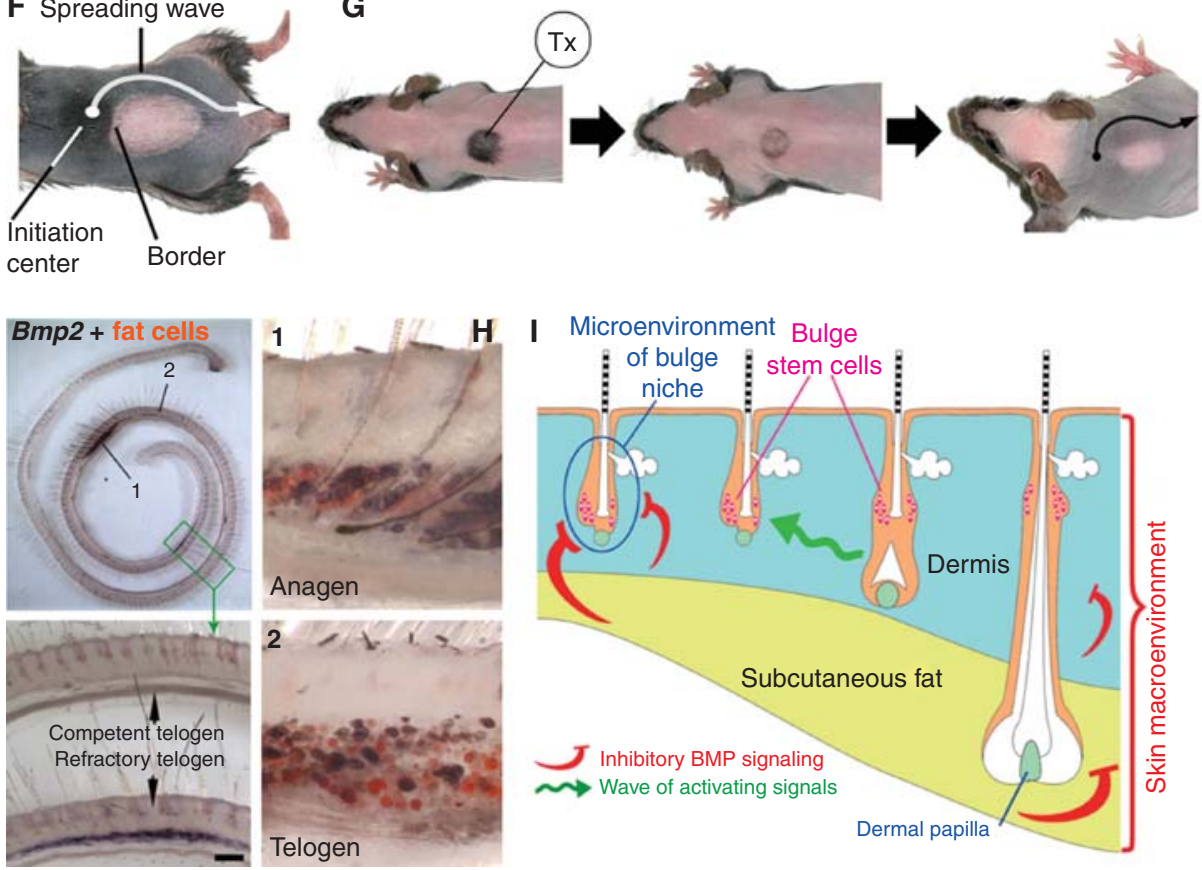

H

I Microenvironment Bulge

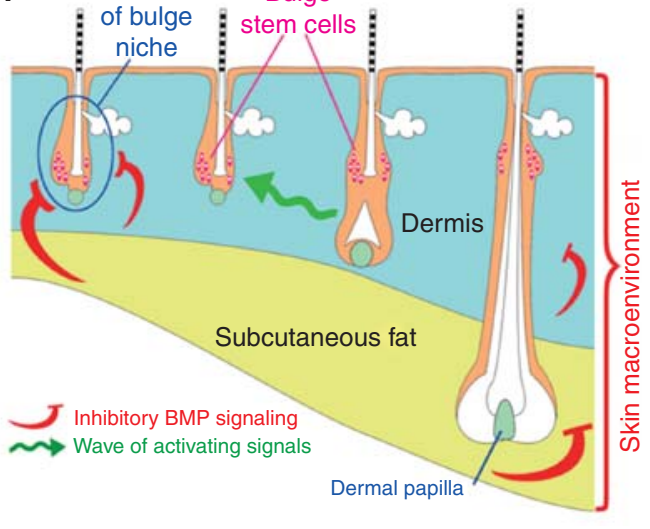

Figure 1. Modulation of the hair regenerative cycles by extrafollicular macroenvironmental factors. (A) Each HF regenerates in cycles consisting from the consecutive phases of growth (anagen), involution (catagen), and inactivity (telogen) (Plikus et al. 2009). (B) All HFs are distributed on the skin in a nearly geometrically precise gridlike pattern initially established during the embryonic development. $(C-E)$ Many thousands of HFs coordinate their growth cycles within one another and produce dynamic regeneration patterns. (Legend continues on following page.) 
M.V. Plikus and C.-M. Chuong

\section{SIGNALING INTERPLAY BETWEEN HFs AND THEIR MACROENVIRONMENT}

It was generally thought that the duration of the hair cycle resting phase in adult mice is largely random and that the timing of hair SC activation events cannot be predicted. Contrary to that, our recent long-term measurements of spontaneous hair pigmentation pattern dynamics revealed that telogen in adult mice $(>2-$ moold animals that developed mature intradermal adipose tissue) lasts for a minimum of $28 \mathrm{~d}$, or roughly 1 mo. Curiously, after the first month, its duration becomes extremely variable, pointing at some fundamental differences in the way HF SCs behave during the first month of telogen and the period thereafter (Plikus et al. 2008). When we compared the behavior of HF SCs throughout the resting phase, we found that they remain refractory to various types of activating signals during early telogen, yet become competent and easily respond to the very same signals after the first month. For example, when a naturally occurring wave of anagen activation spreads from one HF to the next and reaches a population of HFs in early telogen, their SCs do not become activated and the wave goes around that area (Fig. 1F). A similar phenomenon can be reproduced by experimentally enforcing an extranumerary growth cycle in a small group of refractory telogen HFs with topical cyclosporine A treatment. On completion of cyclosporine A-induced anagen, HFs enter into a new refractory telogen, whereas the surrounding nontreated HFs naturally progress into competent telogen. A spontaneous wave of anagen activation can then effectively spread through the competent telogen skin, while leaving refractory HFs in the cyclosporine-A-treated area unaffected (Fig. 1G). Regenerative response of telogen HFs to plucking injury is significantly different between refractory and competent phases. Competent telogen HFs become quickly activated after plucking of just 50 hairs and generally start to grow after $13 \mathrm{~d}$.

At the same time, refractory telogen HFs respond to the 50 hair plucking injury only after a prolonged delay and it takes on average $42 \mathrm{~d}$ for them to start growing. Similar regenerative delay is observed upon plucking of 200 hairs. Competent telogen HFs start to grow after $9 \mathrm{~d}$, whereas refractory telogen HFs start to grow only after 28 d postplucking (Plikus et al. 2008).

To understand the mechanism that underlies these striking differences in regenerative behavior of telogen HFs, we considered two possibilities. According to the first possibility, SCs become intrinsically nonresponsive to anagen-inducing signals during refractory telogen. Such refractivity can be achieved, for example,

Figure 1. (Continued) In mice ( $C$ is from Plikus and Chuong 2008; reproduced, with permission, from the authors) and rabbits ( $D$ is from Plikus et al. 2011; reproduced, with permission, from the authors), these regeneration patterns can be visualized upon animal shaving as prominent pigmentation patterns, where areas of skin with anagen HFs are dark, whereas areas with telogen HFs are light. These hair cycle-dependent changes in skin pigmentation are possible owing to the phenomenon of the anagen-coupled melanogenesis, in which pigment production becomes restricted exclusively to actively regenerating anagen HFs ( $E$ is from Plikus et al. 2011; reproduced, with permission, from the authors). $(F, G)$ Resting phase of the hair cycle can be further divided into early refractory and late competent telogen. Competent telogen HFs can be easily induced to enter new anagen, whereas refractory telogen HFs do not respond to the same activating signals. A wave of anagenactivating signals spreads unobstructed across the competent telogen skin stimulating all telogen HFs in the process. At the same time, it cannot activate refractory HFs, producing a sharp anagen-telogen boundary. Refractory telogen occurs physiologically ( $F$ is from Plikus et al. 2008; reproduced, with permission, from the authors). It can also be generated by cyclosporine A-induced (Tx) hair growth cycle offsetting ( $G$ is from Plikus et al. 2008; reproduced, with permission, from the authors). $(H)$ Refractory properties of telogen HFs are dependent on the inhibitory bone morphogenetic protein (BMP) signaling in their macroenvironment, which includes Bmp2 signaling from the underlying cutaneous adipose tissue (from Plikus et al. 2008; reproduced, with permission, from the authors). (I) Signaling macroenvironment of HFs integrates inputs from the dermis, cutaneous adipose tissue, and neighboring HFs (from Plikus et al. 2008; reproduced, with permission, from the authors). 
through down-regulation of cell surface receptors and/or intracellular mediators for one or several key signaling pathways. Alternatively, HF SCs remain responsive to activation at all times, but the signaling balance at the level of soluble ligands and antagonists in their immediate environment during refractory telogen is shifted toward inhibitory and away from activating pathways.

To differentiate between these two possibilities, we performed comparative gene expression profiling using a whole mount method that lets us look at the expression differences across the entire skin, from head to tail, all in one sample (Fig. 1H). By focusing our attention on genes with dynamic expression patterns during telogen, we have identified several BMP signaling pathway ligands, $B m p 2$ and $B m p 4$, as candidate regulators of telogen refractivity. Previously, BMP signaling has been implicated as the critical regulator of autonomous hair cycle progression (Botchkarev et al. 2001; Zhang et al. 2006; Kobielak et al. 2007). Intriguingly, we showed that instead of being expressed exclusively within the HF SC niche, BMP ligands were expressed in the surrounding tissues including the interfollicular dermis and skin adipose layer (Fig. 1H). In the context of the entire skin, these dermal and adipose BMPs create broad expression fields, uniting thousands of neighboring telogen HFs into distinct refractory populations (Plikus et al. 2008, 2009).

Therefore, unlike a niche microenvironment that provides targeted, short-range signals to SCs of individual HFs, the dermis and adipose layers send broad long-range signals to many HFs at once, thus constituting their signaling macroenvironment (Fig. 1I). Inhibitory macroenvironmental signaling, rather than intrinsic changes in HF SCs responsiveness, appears to play a critical role in maintaining telogen refractivity. Expression of macroenvironmental Bmp2 and Bmp4 ligands largely dwindles down by the end of the first month of telogen, coinciding with the loss of refractory behavior by HF SCs in our functional experiments. Macroenvironment-mediated telogen refractivity can be cut short from 1 mo to just $6 \mathrm{~d}$ by overexpressing soluble BMP antagonist noggin in the skin of
Macroenvironmental Regulation of Hair Cycling

transgenic Krt14-Noggin mice. Telogen refractivity can also be extended past 1 mo upon intradermal delivery of recombinant BMP4 protein (Plikus et al. 2008, 2009).

Within the skin macroenvironment, cutaneous adipose tissue emerges as the key signaling center. In addition to its BMP-mediated inhibitory effect during refractory telogen, adipose tissue exerts activating effect on HF regeneration during telogen-to-anagen transition. Recently, this activating effect was shown to depend on Pdgfa secreted by a subset of cutaneous preadipocytes (Festa et al. 2011). Curiously, DPs appear to be the primary targets for Pdgfa signaling, suggesting that they amplify and relay activating signals from preadipocytes to HF SCs.

In addition, systemic hormones, such as androgens, estrogens, and prolactin, represent another hierarchical layer in HF SCs regulatory signaling network. Acting systemically, hormonal signaling can modulate and even override paracrine macroenvironmental signaling inputs. This is especially obvious in female mice, which largely halt regeneration of their HFs across the entire skin upon pregnancy and lactation. Thereby, systemic pregnancy-related hormones render all telogen HFs refractory, irrespective of their BMP-mediated refractory and competent status. Following the decrease in prolactin levels upon weaning, all HFs become competent to reenter anagen. As a consequence, often complex hair regeneration patterns become reset and HFs regenerate across the entire skin as one synchronous wave (Plikus et al. 2009). Other systemic hormones or their mediators can similarly modulate hair growth, halting or enhancing it. Indeed, recently prostaglandin D2 emerged as the key mediator of testosterone signaling in androgenic alopecia (Garza et al. 2012).

Taken together, these findings expand traditional boundaries of the functionally relevant SC niche. It appears that the SC niche works as an open-ended system, responding to various short- and long-range signaling inputs, including those from the macroenvironment. Broad, long-range signaling inputs, such as those from the dermis and skin adipose layer, are critical for synchronizing regeneration among thousands 
M.V. Plikus and C.-M. Chuong

of neighboring SCs (Fig. 1I) (Hsu and Fuchs 2012; Plikus 2012).

\section{SELF-ORGANIZING REGENERATIVE WAVE PATTERNS REVEAL STOCHASTIC AND YET COORDINATED STEM-CELL ACTIVATION WHICH CAN BE SIMULATED BY CELLULAR AUTOMATA}

Cyclic BMP signaling in the skin macroenvironment provides the basis for synchronizing multiple HFs in the resting phase. However, for HFs to regenerate, their SCs eventually need to become activated. Our analysis of hair growth pattern dynamics shows that there appears to be two primary mechanisms of HF SC activation. First is the spontaneous activation in each HF, resulting in the formation of new hair growth centers (Fig. 2A). Second is the spreading of activation events from one HF to the next like in a chain reaction, resulting in hair growth waves (Fig. 2B). Both mechanisms appear to be equally important. For example, very rare spontaneous activation events in mice become amplified throughout the entire skin via the signal-spreading mechanism. More frequent spontaneous activation events lead to much more dynamic, fractal-like regeneration patterns as seen in rabbits (Fig. 2C). Thus, efficient hair regeneration across the entire skin appears to arise as the result of balanced inhibitory signaling in the skin macroenvironment and activating signal coupling between neighboring HFs. Working together, both components of this mechanism produce visible patterns of hair regeneration.

To understand the mechanism of SC activation in a HF population, we searched for fundamental principles capable of forming patterns analogous to pigmentation patterns produced during hair regeneration. To this end, we have developed a two-dimensional cellular automata (CA) model, where each element of the model, also known as automaton, represents the SC niche of one HF (Plikus et al. 2011). Each automaton on the two-dimensional grid of the CA model cycles through four consecutive phases, designed to simulate four functionally distinct phases of the hair cycle-refractory and competent telogen, and propagating an auton- omous anagen (Fig. 2D,E). Additionally, each automaton can interact with its immediate neighbors, and, for example, can activate them as long as these neighbors are in competent phase, designed to simulate competent telogen of the hair growth cycle. In the context of this model, each phase can also be assigned with the hypothetical activator/inhibitor signaling profile.

Our CA modeling of hair regeneration predicted that coupling between neighboring $\mathrm{HF}$ SC niches can be equally weakened by either inhibitory pathway ligands or activating pathway antagonists (Fig. 2F). Indeed, expression patterns of Bmp2 and Bmp4 described earlier fit into the expression pattern predicted by the model (Fig. 1H). Additional expression profiling aimed at establishing the identity of the activating pathway, showed that several soluble WNT antagonists, Dkk1 and Sfrp4, also fit into the same pattern. They were expressed high in the dermal macroenvironment of refractory, but not competent telogen skin. Together with the fact that several WNT ligands become highly expressed in early anagen HFs (Reddy et al. 2001), our data supported the notion of WNT being the key signaling pathway that couples SC activation between neighboring early anagen and late telogen HFs (Lowry et al. 2005; Enshell-Seijffers et al. 2010).

Fitting into this picture were also the following experimental results. In Krt14-Wnt7a mice that overexpress canonical Wnt7a ligand throughout the skin epithelia, the duration of refractory telogen shortens from 28 to $12 \mathrm{~d}$. Krt14-Wnt7a mice also form many more spontaneous anagen initiation sites compared with WT mice (Fig. 2G). Additionally, intradermal delivery of beads coated with WNT antagonist Dkk1 can disrupt spreading of the anagen activation wave (Fig. $2 \mathrm{H}$ ), whereas administration of Wnt3a beads alone is sufficient to generate a new anagen initiation event among telogen HFs (Fig. 2I) (Plikus et al. 2011).

However, according to another prediction of our CA modeling, the hypothetical activating pathway should show stochastic behavior in telogen HFs during competent, but not refractory phase. Such stochastic behavior was predicted 

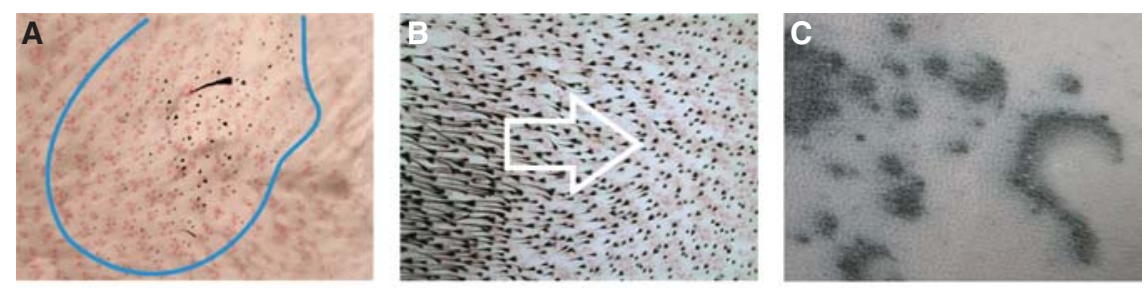

D
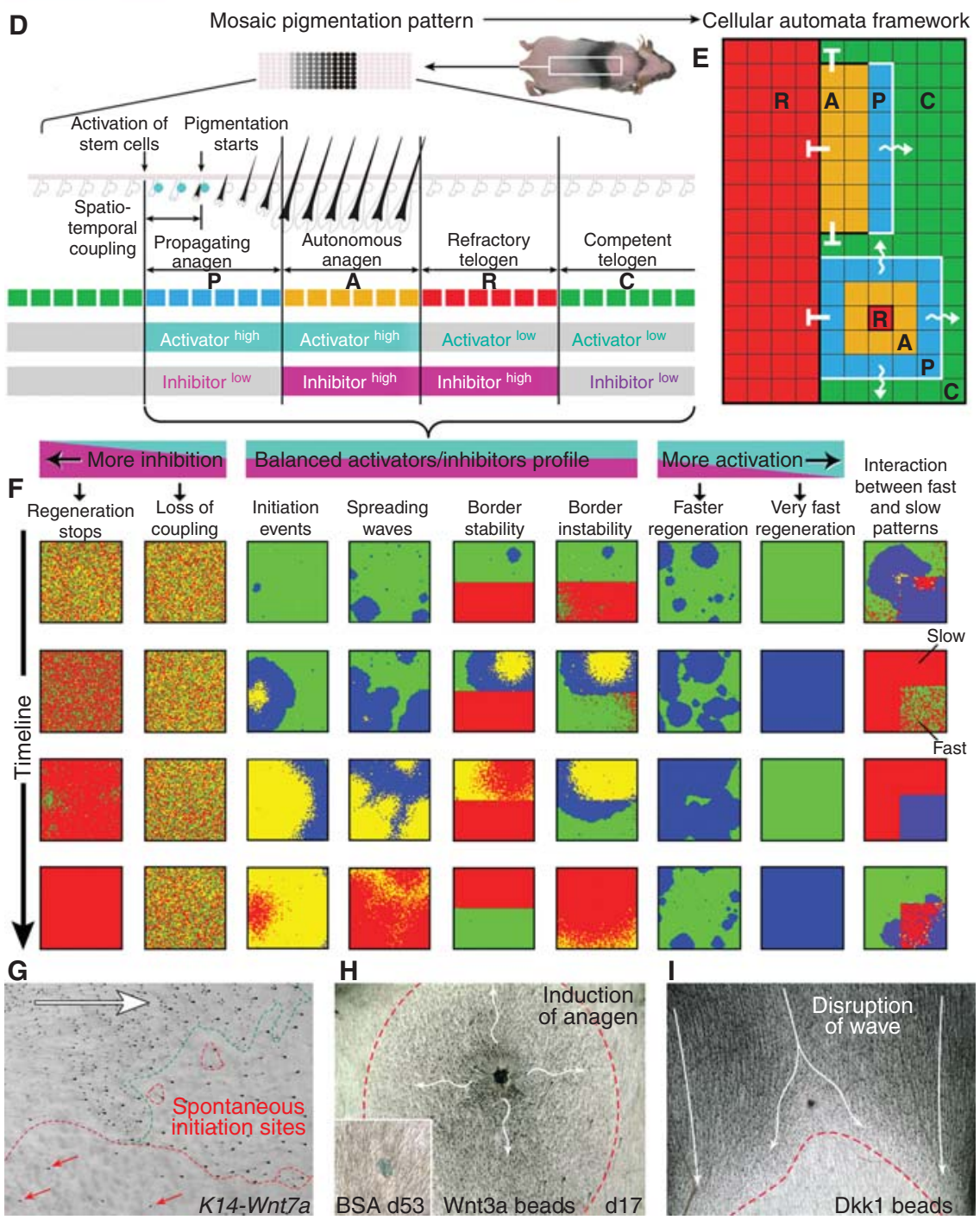

Figure 2. Coupling of hair stem-cell activation among neighboring hair follicles and simulation of coupling behavior with cellular automata. $(A-C)$ Activation of HF SCs and telogen-to-anagen transition can occur spontaneously in each HF or it can spread from one HF to the next in a chainlike reaction. Spontaneous activation events can be visualized as the new hair growth centers within telogen skin $(A)$. Chain reaction activation events appear as spreading hair growth waves $(B)$. The combination of spontaneous activation events and wave spreading results in very dynamic, fractal-like hair regeneration patterns as seen in rabbits (C) (from Plikus et al. 2011; reproduced, with permission, from the authors). (Legend continues on following page.) 
M.V. Plikus and C.-M. Chuong

to form the basis of rare spontaneous anagen activation events in telogen skin. Initially this put in question the role of WNT pathway as the key activator. It was already well established that canonical WNT activation in HF SCs occurs at the time of telogen-to-anagen checkpoint and that such activation event inevitably causes HFs to enter into new anagen. Keeping this in mind, to match the rate of stochastic WNT activation events predicted by the model, the rate of spontaneous anagen initiation would have to be much higher than what is actually observed in mice.

To understand this discrepancy we carefully examined large-scale WNT activation events in the mouse skin using WNT reporter models $B A T-g a l$ and conductin-lacZ. We found that there are additional canonical WNT activation events in telogen HFs localized to DPs, and that they precede WNT activation in HF SCs. These DP WNT activation events occur stochastically during competent, but not refractory telogen phase (Fig. 3A). At any given time point, 5\% of competent telogen HFs display WNT activity in DPs, the rate predicted by our CA model. Also, as predicted by the model, $99.9 \%$ of these DP WNT activation events do not translate into secondary WNT activation events in HF SCs. For this translation to occur, it appears that at least five neighboring telogen HFs have to turn on WNT activity in their DPs simultaneously (Fig. 3C). Considering its stochastic nature, the probability of such an event is very low, yet in agreement with the low probability of spontaneous anagen initiation observed in mice. These results also suggest that WNT-active DPs of telogen HFs must be making some secondary short-range activators, whose concen- trations becomes sufficiently high to activate SCs only when these secondary activators are secreted by several neighboring DPs simultaneously (Plikus et al. 2011).

Indeed, recent studies have identified that canonical WNT activation in DPs is crucial for new anagen initiation and that in response to WNT activation DPs produce secondary activators Fgf-7 and Fgf-10 (Greco et al. 2009; EnshellSeijffers et al. 2010). Taken together, a model of spontaneous anagen initiation emerges in which DPs, which are part of the HF SC niche, produce activating Fgf ligands in response to WNT activation. WNT activation becomes possible only during competent telogen phase, when concentrations of macroenvironmental Dkk1 and Sfrp4 drop sufficiently low to allow for the net balance of WNT ligands and antagonists to transiently and stochastically change in favor of ligands (Fig. 3D). This model shows how both shortand long-range signaling sources can work together to produce a regulatory mechanism of SC activation that has well-balanced robustness and noise-reduction properties (see Fig. 5).

Importantly, this two-step WNT activation mechanism is implicated not only in spontaneous anagen activation, but also in anagen wave spreading. DPs of virtually all telogen HFs assume WNT-active status at the leading edge of the anagen-spreading wave. This all-out DP WNT activation is likely driven by high concentrations of WNT ligands produced by early anagen HFs immediately behind the wave front. This WNT-driven activation of competent telogen HFs by their early anagen neighbors becomes reiterated and can spread across the entire skin like a chain reaction (Fig. 3B) (Plikus et al. 2011). Efficient WNT activation in competent

Figure 2. (Continued) ( $D-F)$ Patterns of hair regeneration can be modeled and studied with the help of a twodimensional cellular automata (CA) model, where each element, also known as automaton, represents one regenerating $\mathrm{HF}$ as it cycles through four functionally distinct phases of refractory $(\mathrm{R})$ and competent telogen $(\mathrm{C})$, and propagating $(\mathrm{P})$ and autonomous anagen $(\mathrm{A})$. Various hair regeneration dynamics can be simulated and predicted based on the same two-dimensional CA framework $(F)$ (from Plikus et al. 2011; reproduced, with permission, from the authors). ( $G-I$ ) Patterns of hair regeneration can be altered by modulating WNT signaling pathway activity. Overexpression of Wnt7a in Krt14-Wnt7a mice shortens refractory telogen and increases the occurrence of spontaneous anagen initiation events $(G)$. Intradermal injection of Wnt3a beads induces a new anagen initiation site $(H)$, whereas injection of WNT antagonist Dkk1 disrupts the normal progression of the physiological hair growth wave (I) (from Plikus et al. 2011; reproduced, with permission, from the authors). 

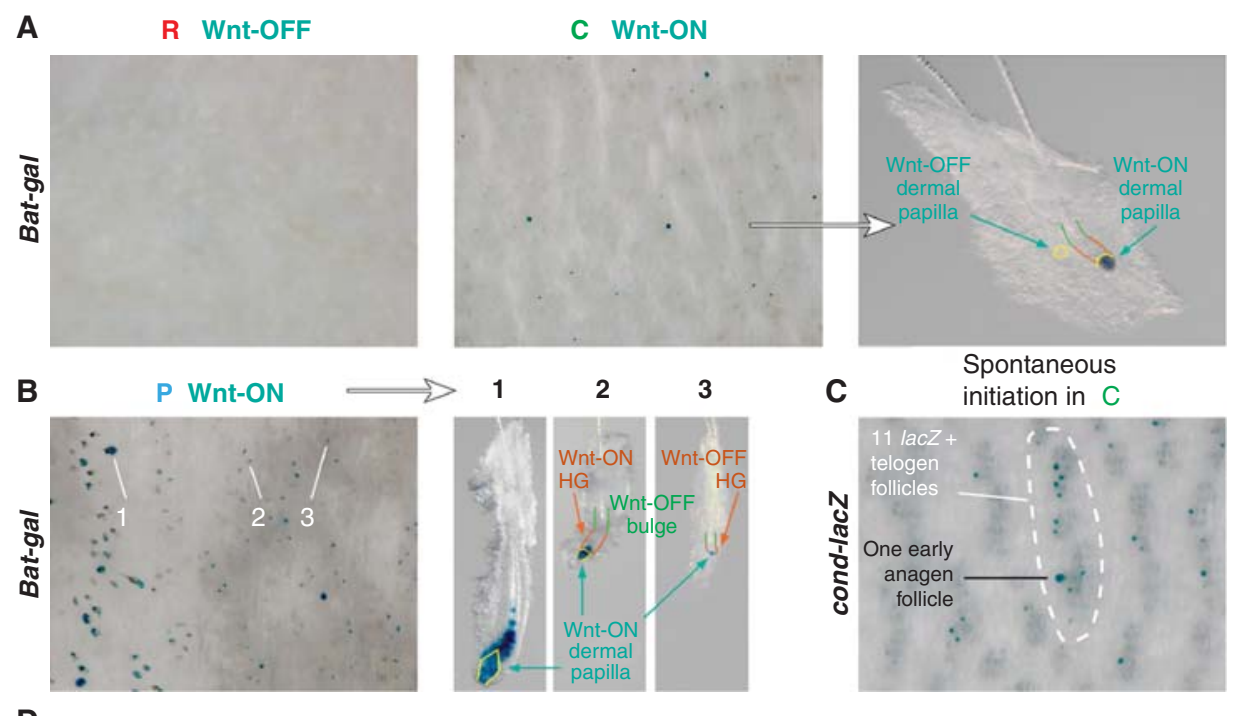

Hair germ
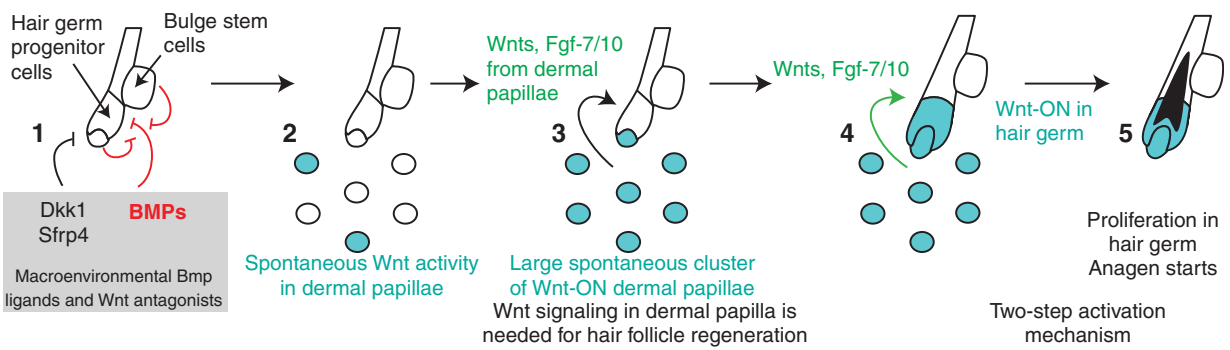

Figure 3. Stochastic and self-organizing regenerative behavior emerges in a population of cross-communicating hair follicles. $(A-D)$ Activation of WNT pathway in DPs of telogen HFs precedes WNT activation in epithelial SCs and initiation of anagen. This early WNT signaling event in DPs occurs in competent, but not in refractory telogen HFs as visualized in BAT-gal and cond-lacZ WNT reporter mice $(A)$. In competent telogen HFs, WNT activation in DPs occurs stochastically and leads to new anagen initiation only when at least five neighboring DPs have turned on WNT activity simultaneously $(C)$. Nearly all telogen DPs in front of the anagen-spreading wave are WNT-active, which translates into $100 \%$ efficient telogen-to-anagen transition by the wave front HFs $(B)$. WNT-active DPs were shown to produce secondary signaling factors such as Fgf-7 and Fgf-10 that facilitate activation of adjacent HF SCs and anagen initiation $(D)$. The schematic drawing in $(D)$ shows the unstable DP activation/stable hair bulge activation/regeneration of one HF/propagation to include the whole HF population. In this process, the random initiation of DP activation is transformed into stable HF activation and robust regenerative hair wave (from Plikus et al. 2011; reproduced, with permission, from the authors).

telogen HFs is also dependent on intrafollicular WNT ligands, whose production in SCs becomes derepressed upon down-regulation of BMP signaling (Kandyba et al. 2013).

\section{COMPLEXITY AND SIMPLICITY OF THE HAIR STEM-CELL SIGNALING NETWORK}

Activation of canonical WNT signaling in epithelial progenitor cells of bulge and hair germ is thought to be critical in the process of telogen HF reactivation (Greco et al. 2009). Considering its importance, this key signaling event in hair regeneration is further modulated and finetuned by additional signaling events acting both in sequence and in parallel. Together they constitute a regulatory signaling network that makes hair regeneration robust, highly adaptable, and noise resistant (Fig. 4). WNT activation in hair germ (Greco et al. 2009) appears to 
M.V. Plikus and C.-M. Chuong

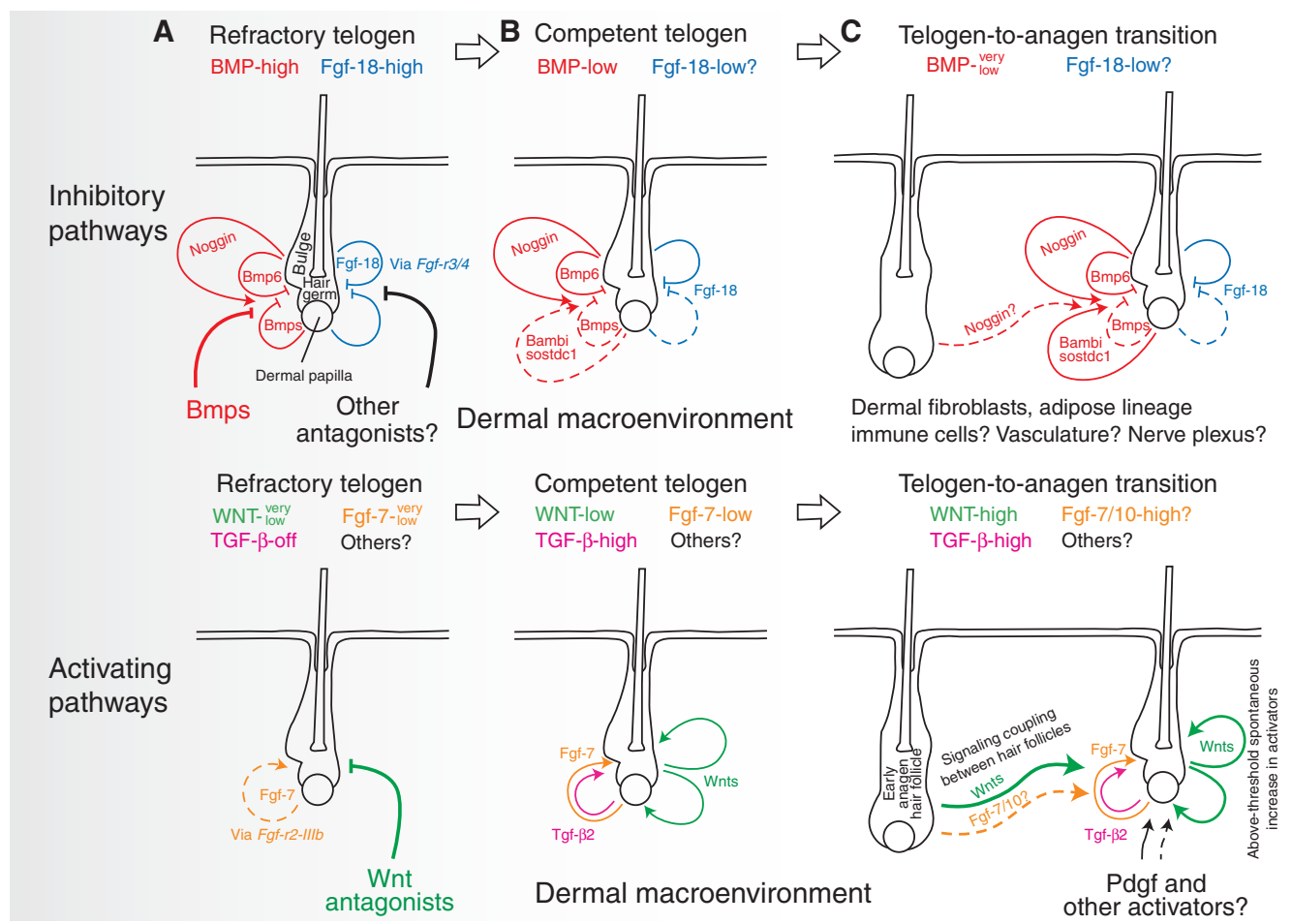

Figure 4. Multilevel signaling inputs are integrated for regulating hair follicle regeneration decision making. Several distinct signaling changes accompany transition from the refractory to competent telogen and from the competent telogen to new anagen. These signaling changes occur across BMP, WNT, FGF, TGF- $\beta$, and, likely, other key morphogenetic pathways (from Plikus 2012; reproduced, with permission, from the author). (A) During the refractory telogen, HFs are exposed to high levels of BMP and Fgf-18 signaling, yet low levels of WNTand Fgf-7 signaling. (B) Transition from the refractory to competent telogen is accompanied by the drop in BMP signaling and the increase in WNT, Tgf- $\beta 2$, and Fgf-7 signaling. This transition is facilitated by the changes in the availability of multiple ligands and antagonists being produced jointly by HFs themselves and their larger dermal macroenvironment. $(C)$ New anagen initiation is driven by the activation of the canonical WNT signaling, first in DPs and then in epithelial SCs. Although the levels of WNT signaling necessary for anagen initiation can likely be achieved spontaneously in the telogen skin, neighboring anagen HFs can act as the source of WNT ligands and facilitate activating signal coupling during anagen wave spreading.

be preceded by WNT activation in adjacent DP (Enshell-Seijffers et al. 2010; Plikus et al. 2011). As mentioned earlier, WNT-active DP secretes Fgf-7 and Fgf-10 ligands that target hair germ progenitors (Greco et al. 2009; Enshell-Seijffers et al. 2010). Although this DP-derived Fgf signaling is essential for anagen activation, it is not completely clear if it provides direct positive feedback, such as by inducing secretion of autocrine WNT ligands by hair germ cells (Greco et al. 2009; Kandyba et al. 2013), or whether it activates them in parallel, in a WNT-independent manner.
BMP and Fgf- 18 signaling provide two complementary inhibitory inputs for bulge and hair germ progenitors during telogen. There are multiple intra- and extrafollicular sources of BMP ligands in the telogen skin. Working together they render telogen HFs refractory to physiological activation (Botchkarev et al. 2001; Kobielak et al. 2007; Plikus et al. 2008). Recent data indicates that the mechanism of BMP-mediated inhibition is at least in part mediated by direct suppression of autocrine WNT ligand expression by HF SCs (Kandyba et al. 2013). Although the strength of inhibitory BMP signal- 
ing appears to be primarily mediated at the level of BMP ligand and antagonist availability, a recent study shows that it can be further regulated at the level of BMP-specific Smads in hair germ progenitors. Tgf- $\beta 2$ starts to be produced by DPs of competent telogen HFs signals to adjacent hair germ cells, where activation of Tgf- $\beta \rightarrow$ phospho-Smad $2 / 3$ signaling dampens $\mathrm{BMP} \rightarrow$ phospho-Smad $1 / 5 / 8$ signaling via a Tmeff1-mediated mechanism (Oshimori and Fuchs 2012). In this respect, Tgf- $\beta 2$ signaling acts as a parallel positive-feedback modulator of anagen initiation.

In addition to BMP, telogen refractivity is maintained by Fgf- 18 signaling. In telogen HFs, Fgf- 18 ligand is secreted largely by keratin 6-positive bulge cells and to a lesser extent by hair germ and DP cells (Blanpain et al. 2004; Greco et al. 2009; Hsu et al. 2011; Kimura-Ueki et al. 2012). Both ablation of keratin 6-positive bulge cells (Hsu et al. 2011) and epithelial deletion of Fgf-18 (Kimura-Ueki et al. 2012) lead to dramatic shortening of refractory telogen and to premature anagen initiation. Opposing effects of Fgf-18 and Fgf-7/10 on hair regeneration can be explained from the perspective of receptor binding affinities. Although inhibitory Fgf-18 preferentially signals via Fgf-r3/4 receptors, activating Fgf-7 signals via Fgf-r2-IIIb receptor (reviewed in Plikus 2012). Interestingly, both BMP and Fgf-18 signaling pathways are required to be operational to maintain telogen refractivity. Disruption of either one of them is sufficient to produce similar short telogen phenotypes (Plikus et al. 2008; Kimura-Ueki et al. 2012). This fact points to the signaling interdependency between BMP and Fgf-18 pathways, details of which are still not understood.

Taken together, instead of being gated by a single master-regulator signaling switch, successive progression of HF SCs from refractory to competent to activated states is regulated by a complex signaling network, comprised of at least BMP, WNT, Fgf-18, Fgf-7/10, Tgf- $\beta$, and Pdgfa pathways. More activators and inhibitors are likely to be found within the context of this network. However, this apparently complex picture can be simplified to a conceptual equilibrium scale of activators and inhibitors (Kandyba
Macroenvironmental Regulation of Hair Cycling

et al. 2013). Basically, HF SCs constantly compare the sum of activator and inhibitor inputs, whichever layer of control it comes from (Chen and Chuong 2012), and make the decision to become activated or to remain quiescent based on the ratio of competing activator/inhibitor activities. Within this framework, new extrafollicular activators or inhibitors can be easily added to modulate the control of hair cycling in response to certain physiological events. This allows HF populations to quickly adapt to changing physiological or pathological conditions.

\section{CONCLUDING REMARKS}

Here we studied collective regenerative behavior in very large populations of SCs using the model of patterned hair growth in shaved mice. From these studies we have learned that periodic spatial patterning during embryonic skin morphogenesis organizes epithelial SCs into discrete clusters, also known as HF SC bulges. In the adult skin, each HF undergoes cyclic episodic regeneration. Together, anatomical prepatterning and cyclic activation of HF SCs provide the basis for patterned regeneration of multiple HFs to occur (Chuong et al. 2012, 2013).

On the body surface of an organism, thousands of HFs can cycle autonomously, synchronously, or in coordination. Our experimental paradigm allows visualizing coordinated hair regeneration waves in living mice. Furthermore, we observe modulation of these hair regeneration waves under different physiological conditions (e.g., pregnancy) (Plikus et al. 2008), in transgenic mice (e.g., Krt14-Noggin Krt14Wnt7a, etc.) (Plikus et al. 2008, 2011), and between different species (e.g., high coupling in rabbits, low coupling in humans) (Fig. 5A) (Plikus et al. 2011).

Based on the novel concept of macroenvironmental regulation of SCs, HF SCs sum up all activator and inhibitor inputs from multilayered signaling networks (Chen and Chuong 2012). Our studies lay down the groundwork for future inquiries into normal and pathologic hair growth behaviors, such as seasonal molting, or androgenetic alopecia. We attempt to 
M.V. Plikus and C.-M. Chuong

A

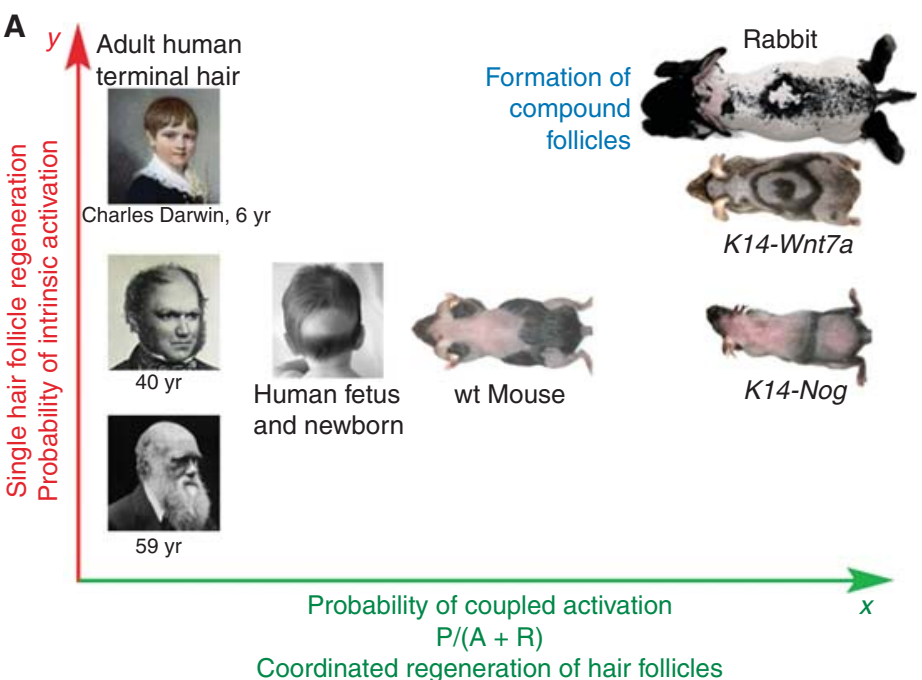

B

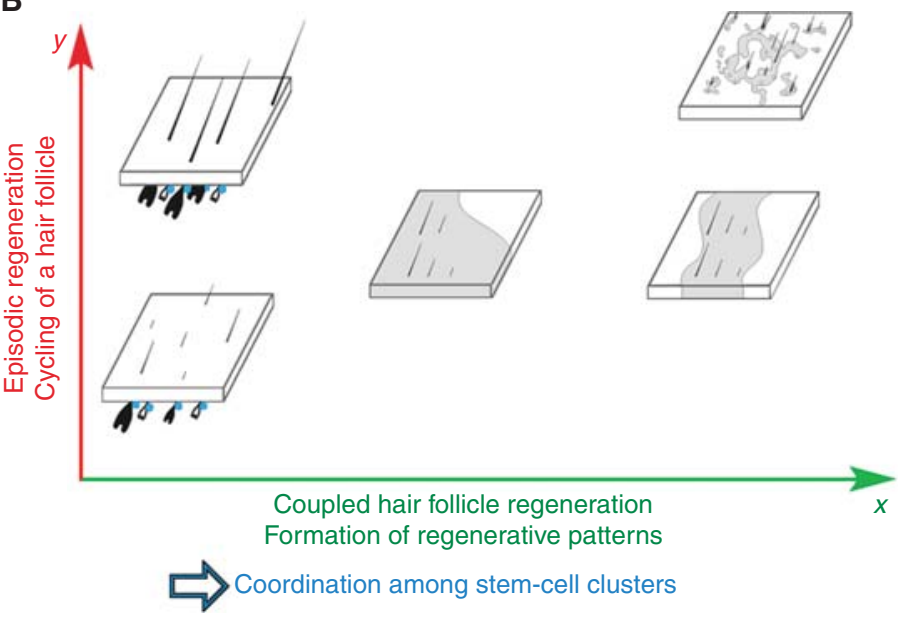

Figure 5. Adaptive evolutionary implications of coupled regenerative behavior in a hair follicle population. (A) Across the surface of the body, populations of HFs show a spectrum of regenerative patterns between different anatomical regions, different ages, or between different species (Cutrone and Grimalt 2005). Selforganizing mechanism of hair regeneration is highly adaptable and depends on the following evolutionary novelties: anatomical clustering of HF SCs (bulge SC clusters), development of cyclic mode of regeneration (hair growth cycle), regulation via macroenvironmental factors, including systemic body hormones. Activation of a single HF is shown in the $y$ axis. Coupling of HFs in the population is shown in the $x$ axis. With an intrinsic mechanism alone, HF regeneration can become easily deficient as seen upon alopecia (toward the left panel). Signal coupling between neighboring HFs enables robust hair regeneration even if the intrinsic probability of SC activation is low (toward the right panel). A wide spectrum of hair regeneration dynamics can be achieved upon modulation of the intrinsic and coupled SC activation mechanisms (from Plikus et al. 2011; reproduced, with permission, from the authors). (B) A more abstractive diagram showing HFs in different cycling states (anagen, telogen). Because of the coupling, HF SCs can become activated by the intrinsic, autonomous hair cycle clock ( $y$ axis). Robust self-initiated cycling is represented by high $y$ value. The level of coupling between neighboring HFs is shown along the $x$ axis, with less or no coupling on the left (human), and higher coupling on the right (from Plikus et al. 2011; reproduced, with permission, from the authors). This hierarchical organization of regulation enables HF populations to adapt to changing physiological or pathological conditions quickly by adjusting skin-wide hair regenerative dynamics. wt, Wild type. 
gain the systems biology level of understanding by identifying the principles that enable coordinated activities in large populations of SCs (Al-Nuaimi et al. 2010). We show that at the higher hierarchical level, large populations of SCs are efficiently managed through stochasticity, coupling, and threshold activation, leading to robust self-organizing regenerative wave, rather than randomness (Fig. 5B).

Beyond hair cycling, we aspire to develop a generic model that can simulate the collective behavior in complex systems consisting of many homologous cycling elements. We use the cellular automata model, in which each automaton (individual HF) cycles through four consecutive phases. The duration of each phase depends on the relative strength of the activator/inhibitor molecular species, which work together to generate phase-switching signaling thresholds (Plikus et al. 2011). Our model successfully simulates a wide spectrum of HF regenerative behaviors. It also shows how significant changes in HF regenerative dynamics can be achieved through simple changes in activator/inhibitor signaling thresholds. This enables large HF populations to quickly adapt to changing physiological or pathological conditions by adjusting skin-wide hair regenerative dynamics.

In summary, the study of HF regenerative behaviors on the two-dimensional space of the skin led us to consider generic principles of patterned biological behaviors. Biological patterns can manifest as spatial periodic patterns (scale, hair, or feather patterns) (Chuong et al. 2013), or as cyclic temporal patterns layered upon preexisting spatial patterns (hair growth waves developing in periodically arranged populations of HFs). We suggest that the principles revealed in our studies may be useful for understanding pattern-forming behaviors in other biological systems ranging in biological complexity from molecular to populational.

\section{ACKNOWLEDGMENTS}

M.V.P. is supported by the Edward Mallinckrodt, Jr. Foundation research grant and the Dermatology Foundation research grant. C.-M.C. is supported by National Institutes of Health NIAMS grants AR60306, 43177, and 47364.

\section{REFERENCES}

Al-Nuaimi Y, Baier G, Watson RE, Chuong CM, Paus R. 2010. The cycling hair follicle as an ideal systems biology research model. Exp Dermatol 19: 707-713.

Arwert EN, Hoste E, Watt FM. 2012. Epithelial stem cells, wound healing and cancer. Nat Rev Cancer 12: $170-180$.

Blanpain C, Lowry WE, Geoghegan A, Polak L, Fuchs E. 2004. Self-renewal, multipotency, and the existence of two cell populations within an epithelial stem cell niche. Cell 118: 635-648.

Botchkarev VA, Botchkareva NV, Nakamura M, Huber O, Funa K, Lauster R, Paus R, Gilchrest BA. 2001. Noggin is required for induction of the hair follicle growth phase in postnatal skin. FASEB J 15: 2205-2214.

Chase HB, Eaton GJ. 1959. The growth of hair follicles in waves. Ann NY Acad Sci 83: 365-368.

Chen CC, Chuong CM. 2012. Multi-layered environmental regulation on the homeostasis of stem cells: The saga of hair growth and alopecia. J Dermatol Sci 66: 3-11.

Chuong CM, Randall VA, Widelitz RB, Wu P, Jiang TX. 2012. Physiological regeneration of skin appendages. Physiology 27: $61-72$.

Chuong CM, Yeh CY, Jiang TX, Widelitz R. 2013. Module based complexity formation: Periodic patterning in feathers and hairs. Wiley Interdiscip Rev Dev Biol 2: 97-112.

Cotsarelis G, Sun TT, Lavker RM. 1990. Label-retaining cells reside in the bulge area of pilosebaceous unit: Implications for follicular stem cells, hair cycle, and skin carcinogenesis. Cell 61: 1329-1337.

Cutrone M, Grimalt R. 2005. Transient neonatal hair loss: A common transient neonatal dermatosis. Eur J Pediatr 164: $630-632$.

Durward A, Rudall KM. 1949. Studies on hair growth in the rat. J Anat 83: 325-335.

Enshell-Seijffers D, Lindon C, Kashiwagi M, Morgan BA. 2010. $\beta$-Catenin activity in the dermal papilla regulates morphogenesis and regeneration of hair. Dev Cell 18: $633-642$.

Festa E, Fretz J, Berry R, Schmidt B, Rodeheffer M, Horowitz M, Horsley V. 2011. Adipocyte lineage cells contribute to the skin stem cell niche to drive hair cycling. Cell 146: $761-771$.

Garza LA, Liu Y, Yang Z, Alagesan B, Lawson JA, Norberg SM, Loy DE, Zhao T, Blatt HB, Stanton DC, et al. 2012. Prostaglandin D2 inhibits hair growth and is elevated in bald scalp of men with androgenetic alopecia. Sci Transl Med 4: $126 \mathrm{ra34.}$

Greco V, Chen T, Rendl M, Schober M, Pasolli HA, Stokes N, dela Cruz-Racelis J, Fuchs E. 2009. A two-step mechanism for stem cell activation during hair regeneration. Cell Stem Cell 4: 155-169.

Hsu YC, Fuchs E. 2012. A family business: Stem cell progeny join the niche to regulate homeostasis. Nat Rev Mol Cell Biol 13: 103-114. 
M.V. Plikus and C.-M. Chuong

Hsu YC, Pasolli HA, Fuchs E. 2011. Dynamics between stem cells, niche, and progeny in the hair follicle. Cell 144: 92-105.

Kandyba E, Leung Y, Chen YB, Widelitz R, Chuong CM, Kobielak K. 2013. Competitive balance of intrabulge $\mathrm{BMP} /$ Wnt signaling reveals a robust gene network ruling stem cell homeostasis and cyclic activation. Proc Natl Acad Sci 110: 1351-1356.

Kimura-Ueki M, Oda Y, Oki J, Komi-Kuramochi A, Honda E, Asada M, Suzuki M, Imamura T. 2012. Hair cycle resting phase is regulated by cyclic epithelial FGF18 signaling. J Invest Dermatol 132: 1338-1345.

Kobielak K, Stokes N, de la Cruz J, Polak L, Fuchs E. 2007. Loss of a quiescent niche but not follicle stem cells in the absence of bone morphogenetic protein signaling. Proc Natl Acad Sci 104: 10063-10068.

Lowry WE, Blanpain C, Nowak JA, Guasch G, Lewis L, Fuchs E. 2005. Defining the impact of $\beta$-catenin/Tcf transactivation on epithelial stem cells. Genes Dev 19: 1596-1611.

Ma L, Liu J, Wu T, Plikus M, Jiang TX, Bi Q, Liu YH, MüllerRöver S, Peters H, Sundberg JP, et al. 2003. "Cyclic alopecia" in Msx2 mutants: Defects in hair cycling and hair shaft differentiation. Development 130: 379-389.

Morris RJ, Liu Y, Marles L, Yang Z, Trempus C, Li S, Lin JS, Sawicki JA, Cotsarelis G. 2004. Capturing and profiling adult hair follicle stem cells. Nat Biotechnol 22: 411-417.

Oshimori N, Fuchs E. 2012. Paracrine TGF- $\beta$ signaling counterbalances BMP-mediated repression in hair follicle stem cell activation. Cell Stem Cell 10: 63-75.

Plikus MV. 2012. New activators and inhibitors in the hair cycle clock: Targeting stem cells' state of competence. J Invest Dermatol 132: 1321-1324.

Plikus M.V Chuong CM. 2008. Complex hair cycle domain patterns and regenerative hair waves in living rodents. J Invest Dermatol 128: 1071-1080.
Plikus MV, Mayer JA, de la Cruz D, Baker RE, Maini PK, Maxson R, Chuong CM. 2008. Cyclic dermal BMP signaling regulates stem cell activation during hair regeneration. Nature 451: 340-344.

Plikus MV, Widelitz RB, Maxson R, Chuong CM. 2009. Analyses of regenerative wave patterns in adult hair follicle populations reveal macro-environmental regulation of stem cell activity. Int J Dev Biol 53: 857-868.

Plikus MV, Baker RE, Chen CC, Fare C, de la Cruz D, Andl T, Maini PK, Millar SE, Widelitz R, Chuong CM. 2011. Selforganizing and stochastic behaviors during the regeneration of hair stem cells. Science 332: 586-589.

Reddy S, Andl T, Bagasra A, Lu MM, Epstein DJ, Morrisey EE, Millar SE. 2001. Characterization of Wnt gene expression in developing and postnatal hair follicles and identification of Wnt5a as a target of Sonic hedgehog in hair follicle morphogenesis. Mech Dev 107: 69-82.

Sick S, Reinker S, Timmer J, Schlake T. 2006. WNTand DKK determine hair follicle spacing through a reaction-diffusion mechanism. Science 314: 1447-1450.

Stenn KS, Paus R. 2001. Controls of hair follicle cycling. Physiol Rev 81: 449-494.

Suzuki N, Hirata M, Kondo S. 2003. Traveling stripes on the skin of a mutant mouse. Proc Natl Acad Sci 100: 96809685.

Tumbar T, Guasch G, Greco V, Blanpain C, Lowry WE, Rendl M, Fuchs E. 2004. Defining the epithelial stem cell niche in skin. Science 303: 359-363.

Whiteley HJ, Ghadially FN. 1954. Hair replacement in the domestic rabbit. J Anat 88: 13-18.

Zhang J, He XC, Tong WG, Johnson T, Wiedemann LM, Mishina Y, Feng JQ, Li L. 2006. Bone morphogenetic protein signaling inhibits hair follicle anagen induction by restricting epithelial stem/progenitor cell activation and expansion. Stem Cells 24: 2826-2839. 


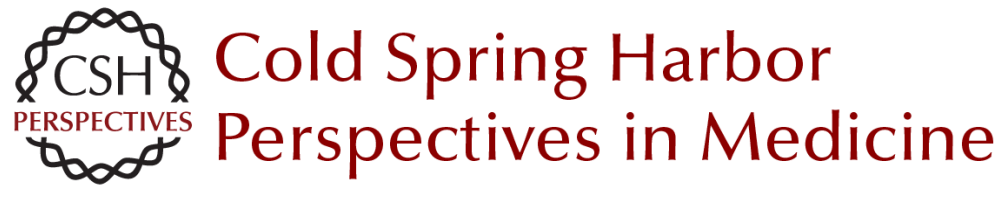

\title{
Macroenvironmental Regulation of Hair Cycling and Collective Regenerative Behavior
}

\author{
Maksim V. Plikus and Cheng-Ming Chuong \\ Cold Spring Harb Perspect Med 2014; doi: 10.1101/cshperspect.a015198
}

\section{Subject Collection The Skin and Its Diseases}

Melanoma: Clinical Features and Genomic

Insights

Elena B. Hawryluk and Hensin Tsao

Wound Healing and Skin Regeneration Makoto Takeo, Wendy Lee and Mayumi Ito

The Dermal Papilla: An Instructive Niche for Epithelial Stem and Progenitor Cells in

Development and Regeneration of the Hair Follicle Bruce A. Morgan

Immunology and Skin in Health and Disease Jillian M. Richmond and John E. Harris

Desmosomes: Regulators of Cellular Signaling and Adhesion in Epidermal Health and Disease Jodi L. Johnson, Nicole A. Najor and Kathleen J. Green

Markers of Epidermal Stem Cell Subpopulations

in Adult Mammalian Skin Kai Kretzschmar and Fiona M. Watt

Psoriasis

Paola Di Meglio, Federica Villanova and Frank O. Nestle

Cell Therapy in Dermatology

Gabriela Petrof, Alya Abdul-Wahab and John A. McGrath
Modeling Cutaneous Squamous Carcinoma

Development in the Mouse

Phillips Y. Huang and Allan Balmain

Natural and Sun-Induced Aging of Human Skin Laure Rittié and Gary J. Fisher

Advanced Treatment for Basal Cell Carcinomas Scott X. Atwood, Ramon J. Whitson and Anthony E. Oro

Epidermal Polarity Genes in Health and Disease Frederik Tellkamp, Susanne Vorhagen and Carien M. Niessen

Induced Pluripotent Stem Cells in Dermatology:

Potentials, Advances, and Limitations Ganna Bilousova and Dennis R. Roop

The Genetics of Human Skin Disease Gina M. DeStefano and Angela M. Christiano

p53/p63/p73 in the Epidermis in Health and Disease

Vladimir A. Botchkarev and Elsa R. Flores

Diversification and Specialization of Touch

Receptors in Skin

David M. Owens and Ellen A. Lumpkin

For additional articles in this collection, see http://perspectivesinmedicine.cshlp.org/cgi/collection/ 